\title{
Teses e dissertações em Ciência da Informação: a multidisciplinaridade não revelada na avaliação da produção científica $^{3}$
}

\author{
Theses and dissertations in Information Science: unrevealed \\ multidisciplinarity in the evaluation of scientific production
}

Daisy Pires NORONHA ${ }^{2}$

Asa $\mathrm{FUJINO}^{3}$

\section{RESUMO}

Reflexão sobre o perfil da comunidade de pesquisadores em Ciência da Informação na ECA/USP, que emerge das análises de indicadores de formação e titulação do corpo docente dos programas de pós-graduação, em relação ao perfil temático das dissertações e teses orientadas. As linhas e os grupos de pesquisa cadastrados no CNPq são elementos de referência na categorização temática da produção científica da área. Partindo da hipótese de que ementas são elaboradas para acomodar interesses heterogêneos de pesquisadores e não refletem a produção da área, foi realizado estudo para analisar o conteúdo temático e objetos de análise das orientações efetuadas de 1999-2004 para avaliar sua pertinência em relação às linhas e grupos de pesquisa. Os resultados mostram uma gama de trabalhos de pesquisa de corpos teóricos diferentes, com novos temas incluídos no bojo da produção acadêmica que confirmam a multidisciplinaridade presente no programa mas, normalmente, não revelada plenamente nos estudos de avalição dessa mesma produção, em função das categorias utilizadas para análise e apresentação de resultados.

Palavras-chave: produção científica; teses; dissertações; Ciência da Informação; avaliação.

\footnotetext{
ABSTRACT

The study discusses the researchers' community profile in Information Science at ECA/USP, that emerges from the analyses of indicators about formal education of the graduate programs' research members in relation to the thematic profile of their dissertations and theses. The department lines of research and the research groups registered at CNPq are referential elements in the evaluation of the scientific production of the area. Working

1 Comunicação apresentada ao VII ENANCIB, Marília, 19-22 novembro 2006.

2 Doutora em Saúde Pública, Universidade de São Paulo, USP, Brasil. Docente, Escola de Comunicações e Artes, Departamento de Biblioteconomia e Documentação, Universidade de São Paulo, USP. Av. Prof. Lucio Martins Rodrigues, 443, 2. Andar, Butantan, 05508-900, São Paulo, SP, Brasil. Correspondência para/Correspondende to: D.P. NORONHA. E-mail: <daisynor@usp.br>.

3 Doutorado em Ciências da Comunicação, Escola de Comunicações e Artes, Universidade de São Paulo, USP, Brasil. Docente, Escola de Comunicações e Artes, Departamento de Biblioteconomia e Documentação, Universidade de São Paulo, USP. Av. Prof. Lucio Martins Rodrigues, 443, 2. Andar, Butantan, 05508-900, São Paulo, SP, Brasil. E-mail: <asfujino@usp.br>. Recebido em 1 1/2/2008 e aceito da publicação em 14/7/2008.
} 
under the hypothesis that the descriptions of the group's activities are elaborated to accommodate the researchers' diverse interests and don't reflect the production of the area, a study was undertaken to analyze the thematic content of the dissertations/theses from 1999 to 2004, for the evaluation of their pertinence to the lines of research and research groups. The results show an unrevealed multidisciplinar academic production in the bibliometric studies, especially quantitative results and the negative impact of those descriptions on the analysis of the production of the area itself, since the production of the graduate program includes a variety of research works from different theoretical fields.

Keywords: scientific production; theses; dissertations; Information Science; evaluation.

\section{INTRODUÇÃO}

O início da década de 1970 foi marcado pela investida na qualificação dos recursos humanos como base para o desenvolvimento da Ciência e Tecnologia (C\&T) brasileira. Com a reforma universitária instituída em 1968 foram criados os cursos de pós-graduação níveis mestrado e doutorado - voltados não só para a qualificação dos docentes como, principalmente, para o fortalecimento das atividades de pesquisa científica, com a produção de novos conhecimentos e fortalecimento de outros.

A instalação dos cursos de pós-graduação em Ciência da Informação (Cl) deu-se nos anos de 1970, com a criação do primeiro curso em nível de Mestrado, no Instituto Brasileiro de Informação Científica e Tecnológica (IBICT) em parceria com a Universidade Federal do Rio de Janeiro (UFRJ); e outros, instalados na mesma década, nas Universidade de São Paulo (USP) em 1972; Universidade Federal de Minas Gerais (UFMG) em 1976; Universidade Federal da Paraíba (UFPb) em 1977, Pontifícia Universidade Católica de Campinas (PUC-Campinas) em 1977 e Universidade de Brasília (UnB) em 1978 (Poblacion, 1993). Após 20 anos do último mestrado criado, foram credenciados pela Coordenadoria de Aperfeiçoamento de Pessoal do Ensino Superior (CAPES) outros três cursos: na Universidade Estadual Paulista Júlio de Mesquita Filho (UNESP) em 1998; na Universidade Federal da Bahia (UFBA) em 1998; e na Universidade Federal de Santa
Catarina (UFSC) em 2003. Em nível de doutorado, o início se dev em 1980, com o primeiro curso na USP; e somente 12 anos mais tarde, é que a área foi contemplada com outros três cursos nas universidades: IBICT/UFRJ (1992); UFMG e UNB (1997) e, mais recentemente, UNESP (2004). Todos esses cursos, stricto sensu, foram devidamente credenciados pela CAPES 4 . Os Programas de Pós-Graduação em Ciência da Informação (PPGsCl), respondendo aos desafios propostos para engrandecimento da área, passam a priorizar o aprimoramento de recursos humanos para o ensino e a pesquisa.

\section{A CONSTITUIÇÃO DO CORPO DOCENTE DOS PPGsCl}

A formação e a titulação dos docentes dos PPGsCl passaram por situação transitória até a consolidação de massa crítica com titulação de doutores provenientes de diferentes áreas. Para os primeiros cursos de pós foram convidados professores titulados em diferentes áreas do conhecimento e, principalmente, renomados especialistas estrangeiros (Pinheiro, 1999/ 2000) devido à escassez de professores titulados na área da $\mathrm{Cl}$. Essa situação garantiu a multidisciplinaridade da área que se fortaleceu, posteriormente, com a mescla de especialização na titulação dos docentes dos programas.

Os primeiros seis PPGsCl (IBICT/UFRJ, USP, UFMG, PUC-Campinas, UFPB, UnB) foram constituídos

\footnotetext{
4 Destacam-se as seguintes considerações:

a) Até o ano de 2005, a Cl na pós-graduação da USP era tida como Área de Concentração junto ao Programa de Pós-Graduação em Ciências da Comunicação da Escola de Comunicações e Artes (PPGCl/ECA). A partir de 2006, passa a se constituir em Programa de Pós-Graduação em Ciência da Informação, na mesma unidade.

b) O Programa da UFPB, em fase de reformulação desde 2002, foi recredenciado pela CAPES, em julho 2006.
} 
por docentes titulados como mestres e doutores (com exceção da USP e UnB que sempre mantiveram doutores em seu quadro docente). A partir de 1999 todo o corpo docente, tanto dos programas mais antigos como os mais recentes, é composto exclusivamente de doutores. Pode-se dizer que a comunidade de pesquisadores em Cl está-se delineando a partir do aumento dos números de PPGs (de seis em 1999 para nove em 2006) e da composição de seu corpo docente, no que se refere a seu crescimento (de 66 em 1999 para 119 em 2006) e na diversificação das áreas na sua formação e titulação.

A diversificação de formação e titulação dos docentes mostra um processo de socialização do conhecimento, o que se constitui em um fator positivo na composição dos PPGs. Essa multidisciplinaridade influenciará os resultados das pesquisas que serão engrandecidos pela experiência e conhecimento dos participantes.

A distribuição do número total de docentes dos PPGsCl, segundo a área de obtenção do título de doutor e o local de obtenção (Brasil ou exterior), identificada na Tabela 1, mostra a natureza interdisciplinar da Ciência da Informação visualizada pelo perfil dos recursos humanos vinculados a esses programas. Os dados encontrados quanto à área e o local de titulação dos docentes dos PPGsCl não apresentam diferenças significativas entre os dois períodos.

Tabela 1. Total de docentes dos PPGsCl, segundo área e local de titulação de doutorado: situação em 1999 e 2006 (jun.).

\begin{tabular}{l|c|c}
\hline Área de titulação & Situação em 1999 & Situação em 2006 \\
\hline Ciência da Informação & $36(54,5 \%)$ & $67(56,3 \%)$ \\
\hline Outras áreas & $30(45,5 \%)$ & $52(43,7 \%)$ \\
\hline Total & $66(100 \%)$ & $119(100 \%)$ \\
\hline Local da titulação de doutor & Situação em 1999 & Situação em 2006 \\
\hline Brasil & $39(59 \%)$ & $29(24,4 \%)$ \\
\hline Exterior & $27(41 \%)$ & $119(100 \%)$ \\
\hline Total & $66(100 \%)$ & Situação em 2006 \\
\hline Pós-Doutorado & Situação em 1999 \\
\hline Ciência da lnformação & $9^{5}$ & $14^{6}$ \\
\hline Outras áreas & $2^{7}$ & $8^{8}$ \\
\hline Total & $11(16,7 \%)$ & $22(19,0 \%)$ \\
\hline
\end{tabular}

Baseada em: Poblácion et al., 2004; Poblácion; Prazeres; Oliveira, 2006.

Em 1999 o corpo docente dos PPGsCl apresentava 54,5\% titulados na área da $\mathrm{Cl}$ (Brasil e exterior) e 45,5\% em outras áreas, titulação obtida no exterior (em administração, computação, comunicação, educação, arquivística, química, engenharia, museologia) e no Brasil (em artes, comunicação, educação, letras, linguística, ciências sociais, saúde pública, sociologia). Esse quadro, em 2006, não apresenta diferenças marcantes. Assim, além dos 67 doutorados em $\mathrm{Cl}$, tanto no Brasil quanto no exterior, 52 titularam-se em outras áreas, no exterior (em museologia, administração, computação, engenharia, sociologa, filosofia, química, história, linguística) e no Brasil (em geografia, educação, comunicação, engenharia, saúde pública, artes, linguística, administração, computação, sociologia, economia, letras). Os países de destino são os mesmos tanto para a titulação de doutorado em $\mathrm{Cl}$ como em outras áreas (EUA, Inglaterra, França e Espanha).

\footnotetext{
5 Inglaterra (4); EUA (2); Alemanha (1); Espanha (1); Canadá (1).

- Brasil (1); Canadá (2); Espanha (2); Inglaterra (2); EUA (3); França (2); Alemanha (1); Portugal (1).

7 Canadá (1); EUA (1).

8 Canadá (2); Inglaterra (1); Espanha (1); França (2); EUA (1); Itália (1).
} 
Assim, o quadro atual mostra um aumento na titulação dos docentes feita no Brasil $(75,6 \%)$ quando comparado à situação de 1999 (59\%). Sem dúvida, a proliferação dos próprios cursos de doutorado tem oferecido condições para que os profissionais da informação obtenham seu título intramuros. Se, por um lado, isso favoreceu o aumento de docentes titulados, por outro deixa escapar a oportunidade para o profissional conhecer novos horizontes e obter experiências com colegas no exterior. No entanto, essa "complementação internacional" poderá (e deveria) ser sanada pelos cursos de pós-doutorado realizados fora do Brasil.

Como se verifica na Tabela 1, o pós-doutorado em 1999 é timidamente representado por 11 docentes $(16,7 \%)$ dos seis PPGsCl. Essa situação, em 2006, embora tenha dobrado em números absolutos (22) ainda continua com pouca representatividade (19\% dos docentes com pós-doutorado). Todos os 11 pósdoutorados feitos até 1999 foram realizados no exterior, sendo um na Espanha, quatro na Inglaterra, dois no Canadá, três nos EUA, e um na Alemanha (Población et al. 2004). O quadro atual ${ }^{9}$ mostra que o pósdoutorado tem sido feito quase que exclusivamente no exterior, com maior procura para o Canadá, EUA e França, seguidos da Espanha e Inglaterra e, com menos frequência, Alemanha, Portugal e ltália. Vale destacar que apenas um pós-doutorado foi realizado no Brasil.

Embora todos os docentes estejam engajados em linhas de pesquisas que abarcam os mais diferentes temas de interesse para a área da $\mathrm{Cl}$, e de onde deveriam emergir os diferentes achados dos estudos realizados, tem-se que o perfil do docente da pós, identificado por sua titulação acadêmica e, sobretudo, por sua formação e pelas linhas de pesquisa em que atua, pode influenciar na orientação aos alunos e na própria produção científica gerada pelas pesquisas realizadas.

Por outro lado, pressupõe-se que no caso brasileiro, a multidisciplinaridade decorrente da participação de especialistas oriundos de diferentes disciplinas não necessariamente caminhou no sentido da interdisciplinaridade que permitiria maior interação entre conceitos, métodos e terminologias, o que possibilitaria a organização de um programa de pósgraduação fundamentado em linhas de pesquisa que teriam influências de diferentes disciplinas mas, como foco de investigação teórico e prático, um mesmo objeto epistemológico. Assim, as linhas de pesquisa dos programas de pós-graduação em Ciência da Informação, bem como os grupos de pesquisa cadastrados nos órgãos de fomento à pesquisa, não correspondem, em sua totalidade, à realidade das pesquisas desenvolvidas sob seu abrigo.

Desse modo, faz-se necessário não apenas conhecer se os estudos orientados pelos docentes dos PPGs condizem com os propósitos das linhas de pesquisa onde se engajam, como também saber até que ponto a sua formação e titulação podem interferir na definição das temáticas das dissertações e teses orientadas, na seleção do objeto de análise e na produção científica gerada e divulgada nos diferentes veículos de publicação. Um elo coerente entre esses pontos - titulação docente, linha de pesquisa e produção gerada - permitirá uma consolidação interdisciplinar mais efetiva dos programas de pósgraduação.

Neste sentido, com um recorte do universo dos PPGsCl vigente em 2006, representado pelo curso da USP, o presente artigo tem por objetivo analisar o conteúdo temático das dissertações e teses apresentadas, seus objetos de análise e sua pertinência em relação às linhas e grupos de pesquisa dos respectivos orientadores. Complementarmente, objetiva identificar como a formação do corpo docente influi na organização temática atual do programa.

\section{Características das Linhas e Grupos de Pesquisa do PPGCI/USP}

Os docentes do PPG da USP na área de concentração Ciência da Informação estavam engajados, de 1999 a 2005, em quatro linhas de pesquisa - Ação Cultural, Análise Documentária; Geração e Uso da Informação, e Informação, Comunicação e Educação. Com a constituição do programa específico de Ciência da Informação, essas quatro linhas foram substituídas por duas - Acesso à Informação e Mediação e Ação Cultural - que abarcam, respectivamente, 13 e quatro docentes.

9 Dados extraídos do currículo dos docentes, na plataforma Lattes/CNPq, em julho de 2006. 
Essas linhas são sinalizadoras dos projetos de pesquisa desenvolvidos e servem de referência para os candidatos aos cursos de pós-graduação que esperam buscar orientação condizente com as temáticas componentes das ementas pertinentes a cada uma das linhas. Por outro lado, servem também de referência para categorização dos resultados da produção científica do corpo docente que influirá na avaliação do programa por organismos externos, a exemplo da CAPES.

É sabido que, além das linhas de pesquisa onde são engajados, os docentes dos PPGs desenvolvem suas investigações em grupos voltados a estudos em áreas específicas da especialidade (Oliveira, 2005). Muitos dos trabalhos desenvolvidos nesses grupos estão em consonância com as temáticas das linhas de pesquisa. No entanto, é comum identificar pesquisas nem sempre condizentes com as linhas de pesquisa oficiais, seja por tratarem de desdobramentos da temática geral, seja por extrapolarem os limites da própria linha ou por serem essas linhas inadequadas como categorias de análise, uma vez que não foram concebidas para tal finalidade.

Em 1999, os docentes do PPGCl-USP participavam de seis Grupos de pesquisa - três envolvendo docentes da linha Geração e Uso da Informação; um da linha Análise Documentária, um da linha Ação Cultural e um da linha Informação, Comunicação e Educação, contando também com a participação de pesquisadores extramuros. Do PPGCl/ USP são cadastrados, no censo de 2004 do Conselho Nacional de Pesquisa (CNPq), quatro Grupos de Pesquisa (Grupo Temma, Design de Sistemas Virtuais, Núcleo de Produção Científica e História em Quadrinhos), com a participação de docentes pertencentes às linhas de pesquisa Análise Documentária e Geração e Uso da Informação. Na base corrente de 2006 do CNPq, no mês de julho, pelo site da instituição, consta o registro dos Grupos Núcleo de Produção Científica e Infoeducação (Población; Prazeres; Oliveira, 2006). Essa diversificação no quadro dos grupos de pesquisa da ECA pode refletir a necessidade de uma consolidação na sua constituição. Deve-se considerar também a possibilidade de os docentes do curso de Biblioteconomia e Documentação estarem participando de atividades de pesquisa realizadas em outros grupos vinculados a outros programas de pós-graduação - nacionais ou do exterior
- e cujas temáticas não sejam objeto de estudos nesse programa ou, ao contrário, que as atuais linhas sejam insuficientes para refletir as pesquisas desenvolvidas no âmago desse programa.

Nesse contexto, considera-se relevante e pertinente conhecer as dissertações e teses orientadas por esse corpo docente e compará-las com as temáticas que são propostas nas linhas e grupos de pesquisa, as quais servem de parâmetro para estudos bibliométricos desses grupos. Partiu-se da hipótese de que ementas são elaboradas para acomodar interesses heterogêneos de pesquisadores do programa com reflexo negativo no retrato da realidade da produção temática da área, quando analisadas de forma agrupada e pelos métodos quantitativos tradicionais, por isso inadequadas para refletir a multi ou interdisciplinaridade da área. Assim, foi realizado estudo para analisar o conteúdo temático e os objetos de análise das orientações efetuadas na área da Ciência da Informação na ECA/USP, com o objetivo de avaliar sua pertinência em relação às linhas e grupos de pesquisa e eventuais pesquisas, cujos objetos de análise extrapolam as atuais categorias de análise. Essa amostra representa um recorte no universo dos programas de pós-graduação em $\mathrm{Cl}$ no país, por ser um dos mais antigos na formação de mestres e pioneiro na formação de doutores e poderá servir para subsidiar estudos sobre o perfil dos pesquisadores e dos temas em Ciência da Informação no Brasil.

\section{PROCEDIMENTOS}

Universo de estudo: Foram analisadas as dissertações e teses orientadas pelos docentes do Departamento de Biblioteconomia e Documentação cadastrados nas linhas de pesquisa do programa de pós-graduação, na área de concentração de Ciência da Informação do Programa de Pós-Graduação em Comunicações da ECA/USP e defendidas no período de 1999 a 2004, por representar o período de consolidação das linhas e grupos de pesquisa. $\bigcirc$ ano de 2005 foi descartado em função de ser um ano em que o programa se encontrava em processo de transformação para início de novo programa específico em Informação e Cultura.

Coleta e análise de dados: Cada documento foi submetido à análise de conteúdo, visando indentificar 
- tema principal e objetos de análise - teórico e prático - do pesquisador/aluno. Os documentos foram categorizados numericamente dentro das linhas de pesquisa, com suas respectivas ementas que apresentam uma síntese das preocupações teórico-metodológicas da linha, e dos respectivos orientadores. Foi elaborado também estudo comparativo das orientações efetuadas entre as diversas linhas, identificando os temas e objetos de análise mais frequentes. Posteriormente procedeuse à análise comparativa entre tema orientado e formação do orientador visando identificar possíveis influências de sua formação na definição dos temas e, sobretudo, se o objeto de análise - teórico ou prático da pesquisa orientada corresponde aos objetos de pesquisa tradicionalmente incluídos no campo da Ciência da informação.

\section{RESULTADOS PRELIMINARES}

Foi analisado o universo total dos 76 documentos produzidos no período de 1999 a 2004, sendo $27(35,5 \%)$ teses de doutorado e 49 (64,5\%) dissertações de mestrado.

Levando-se em conta a produção de teses e dissertações no período, em relação às linhas de pesquisa dos orientadores (Quadro 1), verifica-se equilíbrio entre as linhas ao longo do período, com variações a cada ano, com exceção da linha de Informação, Comunicação e Educação, mas com acúmulo por orientador, notadamente no ano de 2004. Esse acúmulo é explicado pelo fato de tratar-se de pesquisas de doutorado que necessitam de maior tempo de maturação e que dependem de credenciamento do docente junto ao programa de pós. Por outro lado, o fato de a linha de Informação, Comunicação e Educação manter produção às vezes equilibrada mas, no geral menor do que as demais linhas, explica-se pela circunstância de a linha contar com apenas um orientador ao longo do período.

Em relação aos aspectos temáticos das dissertações e teses, a análise mostra uma diversidade bastante significativa nas várias linhas, ao mesmo tempo em que mostra alguns temas, sob perspectivas e facetas diferenciadas, permeando todas as linhas. É o caso das pesquisas que têm a informática ou as ferramentas eletrônicas, as tecnologias de informação e comunicação ou os processos gerenciais, além das relações homemmáquina, como objeto de estudo e suas relações sociais com a cultura, educação e comunicação. a) Na linha de Ação Cultural, as pesquisas realizadas mostram um leque bastante grande dos recortes teóricos, objetos e métodos de estudo, com forte ênfase nas pesquisas relativas a museologia, incluindo discussões metodológicas sobre o processo de concepção de exposições, conservação e restauro de acervos. Também abrangem questões relativas a manifestações culturais, reconhecidas como ações culturais, tais como coro cênico e comerciais de TV. Outro aspecto que vale mencionar é a preocupação com a ação cultural enquanto agente de mudança social, notadamente na contribuição ao desenvolvi-mento sustentável de pequenas comunidades ou na melhoria da qualidade de vida na terceira idade. No que se refere às políticas culturais surge, também, a preocupação com as questões relativas à inclusão cultural e respeito à diversidade. Vale destacar que a titulação dos docentes dessa linha, em Artes e Museologia, condiz com as temáticas estudadas: o docente titulado em Ciência da Informação também tem vivência em Museologia. Observa-se que a produção reflete uma relação com a formação acadêmica dos orientadores, complementar à $\mathrm{Cl}$, evidenciando a multidisciplinaridade, mas sem interação que permita estabelecer relações de interdisciplinaridade.

b) Na linha de Análise Documentária, as pesquisas são mais homogêneas do ponto de vista dos recortes teóricos e processos de análise, mas mostram também a diversidade dos objetos de estudo, aí incluindo-se os audiovisuais, arquivos, bases de dados, periódicos eletrônicos, telejornais, ferramentas pedagógicas, informações estatísticas e sociais. Observa-se, também, preocupação com discussões epistemológicas e a busca das interfaces com a linguística, a terminologia e a comunicação. Por outro lado, mostra, também, que temas mais próximos da área de administração e gestão de serviços de informação, tais como políticas nacionais para produção de bases de dados ou políticas de mediação institucionais, acabaram sendo desenvolvidas no âmbito dessa linha, provavelmente para responder a demandas de qualificação de corpo docente interno ou externo, mas com maior preocupação em focar aspectos teóricos da Cl. Docentes com formação em Biblioteconomia e Linguística compõem o quadro titulado dessa linha. Nessa linha, a interação entre as disciplinas de formação dos orientadores em Linguística ocorre de forma mais integrada, mostrando a multidisciplinaridade caminhando mais no sentido da interdisciplinaridade. 
Quadro 1. Ementa das linhas de pesquisa dos docentes do PPGCI/ECA/USP - orientações 1999 a 2004.

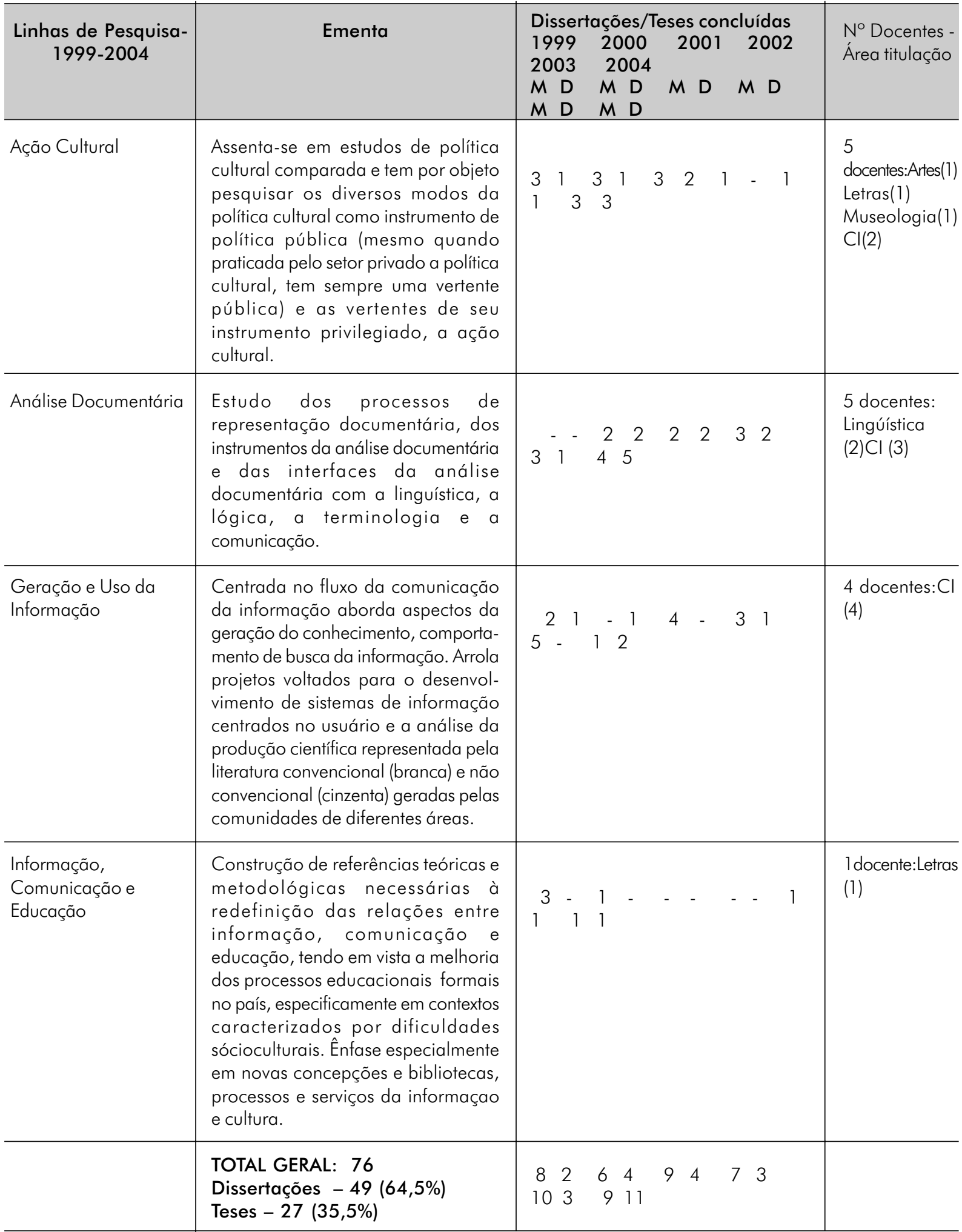


c) Na linha de Geração e Uso da Informação, as pesquisas mostram maior heterogeneidade tanto dos objetos, quanto dos processos de análise, embora maior homogeneidade nos recortes teóricos da $\mathrm{Cl}$. Observa-se forte ênfase nos estudos relativos à comunicação científica e à avaliação da produção científica, em estudos sobre usuários e comportamentos de busca mas, também, estudos sobre gestão de bibliotecas e serviços de informação. Discussões sobre educação a distância, information literacy, metadados, intranets surgem também no esteio das discussões sobre gestão do conhecimento ou das comunidades virtuais. Outro aspecto digno de menção são os estudos que têm como objeto as histórias em quadrinhos. Nesse caso, trata-se de docente pertencente ao quadro de professores do programa de Ciência da Informação mas, também, credenciado no programa de Comunicações. Tal fato é justificado, pois a linha reúne docentes titulados em Ciência da Informação, com interfaces na área de Comunicações, Informática e Administração e por esse motivo acaba abrangendo estudos mais variados. Nessa linha, embora a formação dos pesquisadores seja homogênea e se concentre na $\mathrm{Cl}$, o aporte da Comunicação e da Informática faz-se de modo a complementar os estudos, ressaltando aspectos multidisciplinares. d) Na linha Informação, Comunicação e Educação, as pesquisas mostram maior homogeneidade dos objetos de estudo, normalmente focados nas novas formas de pensamento a apropriação da informação, visando à produção de sentidos, nos espaços educativos ou nos estudos sobre memória e sociedade. Nesse sentido, as pesquisas mostram preocupação com as práticas de leitura e a inserção do lúdico na relação com o público infanto-juvenil e na construção de ambientes interativos como estratégia educativa. Também mostram a preocupação com os aspectos sociais presentes nos projetos que relacionam memória e terceira idade. Toda essa gama de informação esteve sob a orientação de um docente titulado em Letras mas, com maior interação com a Cl.

Quanto aos grupos de pesquisa, por se tratarem de grupos que reúnem pesquisadores de várias instituições, a análise foi realizada somente no intuito de relacionar as pesquisas orientadas por pesquisadores do programa de pós-graduação em Ciência da Informação da ECA/USP e as ementas de tais grupos, para verificar eventual inconsistência com as linhas de pesquisa e pesquisas orientadas (Quadro 2).

Quadro 2. Ementa dos Grupos de pesquisa dos docentes do PPGCI/ECA/USP - Censo 2004.

\begin{tabular}{l|l|c|c}
\hline \multicolumn{1}{c|}{$\begin{array}{c}\text { Grupos de } \\
\text { Pesquisa-2006 }\end{array}$} & $\begin{array}{c}\text { Ementa } \\
\text { PPGCl docentes do } \\
\text { participantes }\end{array}$ & $\begin{array}{c}\text { Linhas de } \\
\text { pesquisa s } \\
\text { (1999-2004) }\end{array}$ \\
\hline Grupo Temma & $\begin{array}{l}\text { Atua na construção de conhecimentos } \\
\text { relacionados à organização da informação, em 3 } \\
\text { eixos de ação: processo da análise documentária } \\
\text { (indexação); análise da função comunicacional } \\
\text { dos produtos gerados (resumos, índices); variáveis } \\
\text { intervenientes na construção de vocabulários } \\
\text { controlados. }\end{array}$ & $\begin{array}{c}\text { Análise } \\
\text { Documentária }\end{array}$ \\
\hline Design de Sistemas & $\begin{array}{l}\text { Oferecer metodologias, estudos e discussão } \\
\text { sobre análise e compreensão de problemas } \\
\text { práticos sobre o desenvolvimento de siste-mas } \\
\text { virtuais à luz de teorias orientadas a partir da } \\
\text { lente do usuário da informação. Desenvolver e } \\
\text { testar metodologias de modelagem conceituale } \\
\text { de design de sistemas virtuais centrado no usuário, } \\
\text { a partir de variadas abordagens técnicas e } \\
\text { ferramentas oriundas das áreas da Ciência da } \\
\text { Informação e lnteração Homem-Computador sob } \\
\text { suas diferentes vertentes }\end{array}$ & 2 \\
\hline
\end{tabular}




\begin{tabular}{l|c|c|c}
\hline \multicolumn{1}{c|}{$\begin{array}{c}\text { Grupos de } \\
\text { Pesquisa-2006 }\end{array}$} & Ementa & $\begin{array}{c}\text { No docentes do } \\
\text { PPGCl participantes }\end{array}$ & $\begin{array}{c}\text { Linhas de } \\
\text { pesquisa s } \\
\text { (1999-2004) }\end{array}$ \\
\hline $\begin{array}{l}\text { Núcleo de Produção } \\
\text { Científica }\end{array}$ & $\begin{array}{l}\text { Atuar em estudos bibliométricos e cienciométricos } \\
\text { da produção científica da área da Ciência da } \\
\text { Informação ca racterizada pela literatura } \\
\text { convencional e não convencional (cinza). } \\
\text { Concentrar estudos em segmento da comunidade } \\
\text { científica da área, centrada nos docentes dos } \\
\text { Programas de Pós-Graduação brasileiro. }\end{array}$ & $\begin{array}{c}\text { Geração e } \\
\text { Uso da } \\
\text { Informação }\end{array}$ \\
\hline História em & $\begin{array}{l}\text { Núcleo de pesquisa interdisciplinar com projetos } \\
\text { Qcadêmicos envol-vendo quadrinhos, além de } \\
\text { troca de conhecimentos e informações sobre as } \\
\text { novidades do meio, como lançamentos de } \\
\text { quadrinhos de destaque, bons projetos e iniciativas } \\
\text { diversas. Objetiva preservar a memória do } \\
\text { quadrinho brasileiro, garantindo que as gerações } \\
\text { futuras, bem como pesquisadores e interessados } \\
\text { em geral, tenham conhecimento do que foi } \\
\text { publicado no país nessa área. Busca contribuir } \\
\text { para o estudo dessa produção e sua inserção no } \\
\text { panorama quadrinhístico internacional. }\end{array}$ & 1 & $\begin{array}{c}\text { Geraçãoe } \\
\text { Uso da }\end{array}$ \\
Informação \\
\hline
\end{tabular}

Deste modo, observa-se que o grupo Temma abriga a maior parte dos docentes da linha de pesquisa em Análise Documentária, o Núcleo de Produção Científica, a maior parte dos docentes da linha de pesquisa sobre Geração e Uso, devendo ser mencionados à parte os outros dois grupos: o de Design de Sistemas Virtuais, coordenado por docente da linha Geração e Uso, mas com uma vertente específica de pesquisa sobre sistemas virtuais centrados no usuário. No caso do núcleo de pesquisas em Histórias em quadrinhos (HQ), há apenas um pesquisador do programa de pós analisado, associado a esse grupo.

Nos dois primeiros grupos, não há inconsistências a serem destacadas além das observações já mencionadas na relação entre dissertações/teses e linhas de pesquisa. No caso tanto do grupo de Design de Sistemas Virtuais, quanto o de $H Q$, por tratar-se de faceta já observada, também na análise das linhas de pesquisa, há que se comentar somente a importância de estimular o estabelecimento de relações acadêmicas entre os docentes das linhas de pesquisa dos programas de pós-graduação e aqueles vinculados aos grupos de pesquisa no âmbito das agências de fomento.

\section{CONSIDERAÇÕES FINAIS}

Os dados encontrados mostram algumas questões presentes na inconsistência temática das linhas de pesquisa e dos grupos de pesquisa da área com as teses e dissertações defendidas nos últimos anos, que merecem uma séria reflexão.

As diferenças do perfil do docente com relação à sua formação e titulação acadêmica vêm mostrando uma mudança na abordagem dos estudos realizados nos cursos de PG. Com isso, novos temas estão sendo incluídos no bojo da produção acadêmica em função da ampla diversificação da atuação do corpo docente e do empenho desses programas em atender as demandas sociais e acadêmicas que surgem no contexto da sociedade da informação. Cabe observar que, embora o PPG da ECA conte com corpo docente titulado tanto em Ciência da Informação (na sua maioria) como em outras áreas, nem todos ainda se encontravam, no período, credenciados para orientação de teses de doutorado pelo fato de as normas da USP determinarem um tempo mínimo para esse credenciamento que exige do docente ter ministrado 
disciplinas no programa e ter formado, no mínimo, dois mestres.

A questão da multidisciplinaridade, visível nos dados do perfil do corpo docente e, principalmente no perfil das pesquisas orientadas, pode estar refletindose na fragmentação temática das linhas e dos grupos de pesquisa do PPGCI da ECA/USP, não contribuindo para uma efetiva interação dessas diferentes disciplinas com a Cl. Por outro lado, a necessidade de uma definição e delimitação precisas das linhas de pesquisa, enquanto categorias de análise para estudos de avaliação da produção científica, contrapõe-se à instabilidade característica das áreas em desenvolvimento. Se, por um lado, é necessária flexibilidade para permitir a inserção de novos pesquisadores e novos temas, por outro lado, o uso dessas linhas levam a um retrato que não reflete o estado da arte das pesquisas na área e tampouco propiciam o reconhecimento e disseminação das novas pesquisas e a interação entre pesquisadores com interesses comuns, tão necessária à consolidação das redes sociais presentes informalmente na comunidade científica.

A interação entre disciplinas, para além da justaposição, é necessária para fortalecer o referencial teórico do campo científico, do mesmo modo que as interações interinstitucionais com ações conjuntas dos PPGs; o apoio das agências de fomento à demanda para realização de novos estudos e o aperfeiçoamento profissional são pontos que merecem uma reflexão bastante ampla para a consolidação definitiva da área da $\mathrm{Cl}$.

\section{REFERÊNCIAS}

OLIVEIRA, M. Os grupos de pesquisa em Ciência da Informação: pesquisadores e produção cientifica. In: ENCONTRO NACIONAL DE PESQUISA EM CIÊNCIA DA INFORMAÇÃO-ENANCIB, 6., 2005, Florianópolis. Anais... Florianópolis, 2005. [CD-ROM].

PINHEIRO, L.V.R. Infra-estrutura da pesquisa em Ciência da Informação no Brasil. Revista de Biblioteconomia de Brasília, v. 23/ 24, n.3, p.367-90, 1999/2000. Número especial.

POBLACIÓN, D.A. Investigación y estudios de posgrado em Ciencia de la Información em Brasil: (1970-1985 y 1986-1992). Ciencias de la Información, v.24 n. 1, p.16-21,1993.
POBLACIÓN, D.A. et al. Evolução do perfil do corpo docente dos Programas de Pós-graduação em Ciência da Informação. In: Seminário Nacional de Bibliotecas Universitárias, 13., 2004, Natal. Anais... Natal : UFRN, 2004. CD-ROM.

POBLACIÓN, D.; PRAZERES, A.P.P.; OLIVEIRA, L.C. Evolução dos grupos de pesquisa em Ciência da Informação no diretório do CNPq. In: SEMINÁRIO NACIONAL DE BIBLIOTECAS UNIVERSITÁRIAS, 14., 2006, Salvador. Anais... Salvador, 2006. CD-ROM. 